PACS: $07.85 . \mathrm{Nc} ; 29.50 .+\mathrm{V}$

\title{
BRIEF ANALYSIS OF REAL-TIME DATA COMPUTER REGISTRATION, TRANSMISSION AND PROCESSING METHODS IN NUCLEAR PHYSICS
}

\author{
A. Yefremov, S. Reva \\ V.N. Karazin Kharkiv National University \\ 61022, Kharkiv, 4, Svobody sq., tel/fax 8-057-705-12-47 \\ E-mail: iec-lab@,karazin.ua \\ Received October 23, 2017
}

Structural and principal problems in the field of control equipment for nuclear physics development are examined, in particular, the influence of dead time on the spectrum data aggregation and methods of correcting this influence. The variants of the organization of spectrometric systems, their advantages and disadvantages, the possibility of using different digital interfaces and computer buses are analyzed, and some of existing spectrometric equipment implementations examples are reviewed. The result of the work is an analysis of existing approaches to the equipment for electronic spectrometry organization and development, as well as a recommendation based on the approaches and different technological solutions to achieve the best ratio of performance and versatility is given. The problems that may be encountered in the spectrometric equipment development process and the existing solutions are described. The workability of various operating systems and real-time programming modules to the organization of processing equipment is examined, also the impact on the development-related cost and laboriousness of the opted facilities for solving these problems is examined, and the optimal solutions are proposed.

KEY WORDS: electronic spectrometry, dead time, analog-to-digital converter, computer buses, operating systems

\section{СТИСЛИЙ АНАЛІЗ МЕТОДІВ КОМП'ЮТЕРНОЇ РЕССТРАЦЇ,, ПЕРЕДАЧІ ТА ОБРОБКИ ДАНИХ РЕАЛЬНОГО ЧАСУ У ЯДЕРНІЙ ФІЗИЦІ А.О. Сфремов, С.М. Рева \\ Харківський національний університет імені В.Н. Каразіна 61022, пл. Свободи, 4, тел./факс 8-057-705-12-47}

Розглядаються конструкційні та принципові проблеми в галузі розробки управляючого обладнання для ядерної фізики, зокрема, вплив мертвого часу на побудову спектра та методи корекції цього впливу. Аналізуються варіанти організації спектрометричних систем, їх переваги та недоліки, можливість використання різних цифрових інтерфейсів і комп'ютерних шин, наводиться приклад деяких існуючих реалізацій спектрометричного обладнання. Результатом роботи $\epsilon$ аналіз існуючих підходів до організації та розробки обладнання для електронної спектрометрії, а також рекомендація на основі розглянутих підходів і тих чи інших технологічних рішень для досягнення найкращого співвідношення продуктивності $\mathrm{i}$ універсальності. Описано проблеми, що виникають в процесі розробки спектрометричного устаткування, та існуючі варіанти їх вирішення. Розглянуто можливість застосування різних операційних систем і модулів реального часу для побудови апаратури реєстрації та обробки спектрів, а також вплив обраних засобів вирішення проблеми на вартість $\mathrm{i}$ трудомісткість розробки, запропоновані оптимальні варіанти.

КЛЮЧОВІ СЛОВА: електронна спектрометрія, мертвий час, аналого-цифрові перетворювачі, комп'ютерні шини, операційні системи.

\section{КРАТКИЙ АНАЛИЗ МЕТОДОВ КОМПЬЮТЕРНОЙ РЕГИСТРАЦИИ, ПЕРЕДАЧИ И ОБРАБОТКИ ДАННЫХ РЕАЛЬНОГО ВРЕМЕНИ В ЯДЕРНОЙ ФИЗИКЕ}

\section{А.А. Ефремов, С.Н. Рева}

Харьковский национальный университет имени В.Н. Каразина 61022, пл. Свободы, 4, тел./факс 8-057-705-12-47

Рассматриваются конструкционные и принципиальные проблемы в сфере разработки управляющего оборудования для ядерной физики, в частности, влияние мёртвого времени на построение энергетических спектров и методы коррекции этого влияния. Анализируются варианты организации спектрометрических систем, их преимущества и недостатки, возможность использования различных цифровых интерфейсов и компьютерных шин, приводится пример некоторых существующих реализаций спектрометрического оборудования. Результатом работы является анализ существующих подходов к организации и разработке оборудования задач электронной спектрометрии, а также рекомендация на основе рассмотренных подходов и тех или иных технологических решений для достижения наилучшего соотношения производительности и универсальности. Описаны проблемы, возникающие в процессе разработки спектрометрического оборудования, и существующие варианты их решения. Рассмотрена применимость различных операционных систем и модулей реального времени для организации обрабатывающей аппаратуры, а также влияние выбранных средств решения проблемы на стоимость и трудоёмкость разработки, предложены оптимальные варианты.

КЛЮЧЕВЫЕ СЛОВА: электронная спектрометрия, мёртвое время, аналого-цифровые преобразователи, компьютерные шины, операционные системы.

\section{АКТУАЛЬНОСТЬ ЗАДАЧИ}

При разработке программного обеспечения для обслуживания спектрометрических аналого-цифровых преобразователей (АЦП) авторы статьи столкнулись с задачей выбора структуры аппаратно-программных (C) Yefremov A., Reva S., 2017 
комплексов, методов взаимодействия с аппаратурой, передачи и первичной обработки данных. Использование современных операционных систем с ограниченным доступом к аппаратным ресурсам компьютера, а также отсутствие в компьютерах общего назначения аппаратных интерфейсов промышленного применения создают ряд проблем, связанных с обеспечением надежности и своевременности передачи и обработки информации. Во многих случаях это может приводить к искажению или частичной потере получаемых (обрабатываемых) экспериментальных данных.

Так, например, электронная спектрометрия на базе многоканальных анализаторов с применением аналогоцифровых преобразователей последовательного приближения или АЦП Вилкинсона всегда сопровождается неизбежными погрешностями счёта. В частности, эти просчёты возникают из-за мёртвого времени спектрометрического тракта, когда из-за занятости или каких-либо других причин спектрометр не способен регистрировать поступающие импульсы. Соответственно, каждая часть спектрометрической системы, т.е. измерительный блок, АЦП или устройство обработки информации, вносит свой вклад в мёртвое время измерения. Минимизация и учет мертвого времени является одним из важных условий при решении некоторых научных и прикладных задач ядерной физики. Однако у каждого из отдельных узлов спектрометрического тракта может быть разный тип вносимого мёртвого времени. Различают устройства с мёртвым временем продлевающегося и непродлевающегося типа.

В устройствах с мёртвым временем продлевающегося типа задержки во времени измерения возникают после любого поступившего события и не зависят от того, регистрируется оно или нет. Например, формирующий усилитель может относиться к такому типу устройств. С другой стороны, непродлевающееся мёртвое время не зависит от поступления событий в течение измерительного периода, что характерно, например, для спектрометрических АЦП. Естественно, что различные типы мёртвого времени могут вносить различный вклад в погрешности счёта и даже влиять на форму получаемого спектра [1].

В самом простом случае мы имеем постоянное мёртвое время непродлевающегося типа при постоянной скорости счета детектируемых событий. Такое мёртвое время может быть характерно для систем, задержки в которой связаны с занятостью медленной шины данных с постоянной задающей частотой. В данных условиях мёртвое время влияет лишь на общее время набора спектра с заданной статистической точностью. Однако, в реальности, конечно, это далеко не единственный вариант вклада мёртвого времени в измерительный процесс. Например, характерным для АЦП Вилкинсона вкладом в мёртвое время является зависимость времени процесса измерения сигнала от его амплитуды, так как она напрямую влияет на время полного разряда конденсатора [2]. Также существует проблема с обработкой входных сигналов на измерительном устройстве в условиях множественной диспетчеризации процессов (когда вклад работы операционной системы в занятость процессора может быть достаточно значительным), в частности, это характерно для драйверов или клиентских приложений операционных систем общего назначения.

Данные проблемы, связанные с программно-аппаратной обработкой информации в системах реального времени, и являются предметом рассмотрения в работе. Изложенный в статье материал не является полноценным обзором существующих методов передачи и обработки экспериментальных данных в ядерной физике, а, скорее всего, есть попыткой поиска приемлемого решения частной задачи на основе собственного опыта, аналитических обзоров и публикаций других авторов.

Целью работы является анализ существующих информационных технологий и определение на основе результатов этого анализа наиболее эффективных способов построения компьютеризированных систем реального времени для автоматизации измерений и управления физическими установками.

\section{СПОСОБЫ АППАРАТНО-ПРОГРАММНОЙ ОРГАНИЗАЦИИ КОНТРОЛЬНО-ИЗМЕРИТЕЛЬНЫХ СИСТЕМ РЕАЛЬНОГО ВРЕМЕНИ}

К решению задачи регистрации и обработки спектра с минимальной потерей информации можно подходить с нескольких позиций.

1) Конструкционный подход. Рассматривая спектрометрическую систему с позиции физически взаимодействующих модулей, её можно представить в виде различных вариантов компоновки. Это может быть полноценный спектрометр на базе микропроцессорной системы, исполненный в едином отдельном модуле и подключённый к компьютеру через определённый интерфейс. Либо это может быть только спектрометрический АЦП с контроллером шины, использующий для работы ресурсы компьютера.

2) Концептуальный подход. Очевидно, что программная реализация алгоритмов, процедур ввода-вывода информации и своевременной обработки сигналов — это одна из важнейших частей спектрометрической системы. Соответственно, реализация может осуществляться на базе микроконтроллера, программируемой логической матрицы, или же с помощью универсального компьютера под управлением различных операционных систем.

Рассмотрим различные варианты решения проблемы мёртвого времени, описанной выше.

Экономически, конечно же, целесообразно создать спектрометрический АЦП на плате с контроллером определённой шины, подключать его к детектирующему устройству и к компьютеру, чьи ресурсы и будет использовать измерительное оборудование. При таком подходе есть определённая доля вариативности в 
выборе аппаратных и программных средств реализации. Во-первых, существует множество различных стандартов шин, используемых как в домашних (настольных), так и в промышленных компьютерах (эта же вариативность касается и системы удалённого спектрометрического оборудования). Из распространённых вариантов можно выделить следующие.

Протокол RS-232 (также известен, как последовательный коммуникационный (СОМ) порт). Использование СОМ-порта для коммуникации с устройством имеет свои преимущества и недостатки. Конечно же, логическое программирование интерфейса взаимодействия с последовательным портом довольно простое, даже если речь идёт об операционных системах общего назначения. Из аппаратной реализации требуется только наличие правильно сконфигурированного модуля UART и разъема. Сама шина с физической точки зрения является достаточно неприхотливой, длина соединительного кабеля может составлять десятки метров. Однако, очевидным минусом такой шины является скорость передачи данных, по стандарту ограниченной 115 кбод, что примерно соответствует 70 мкс на передачу однобайтового слова в одну сторону [3].

Шина ISA (Industry Standard Architecture). Универсальная параллельная шина ввода-вывода, системная шина для IBM-совместимых компьютеров. Способна работать на частоте до 8 МГц, приводит к задержке времени в 2-4 мкс на синхронизацию и передачу слова. Данная шина является на текущий день устаревшим стандартом, однако продолжает использоваться в некоторых моделях промышленных компьютеров. Способна поддерживать до 6 подключённых устройств одновременно, а также её расширения вводят технологии DMA и $\mathrm{PnP}$ [4], [5], что упрощает программирование аппаратного управления на логическом уровне. С точки зрения системной компоновки использование данной шины может облегчить как программную, так и аппаратную реализацию спектрометрической системы. Например, модуль АЦП не нуждается в контроллере шины, если нет необходимости использовать технологии DMA или РnP. Однако, с точки зрения системы не будет возможности динамического обнаружения и настройки подключённого оборудования.

Шина PCI (peripheral computer interconnect). Также параллельная системная шина для IBMсовместимых компьютеров, пришедшая на смену шине ISA. Отличается гораздо более высокой пропускной способностью (до 66 МГц при ширине канала в 64 бита) [3], [4]. Данная шина отличается более сложной схемотехникой и арбитражем, дополнительным функциональным и конфигурационным пространством, прерываниями, основанными на передаче сообщений, и многими другими нововведениями. Системная разработка под данную шину может оказаться весьма трудоёмким процессом, а вклад других системных устройств в арбитраж шины может внести в работу оборудования реального времени недетерминированные задержки. Кроме того, РСІ-совместимое устройство нуждается в контроллере шины, так как аппаратное управление обеспечивается сложным механизмом конфигурирования.

Шина PCI Express. Современная системная шина IBM-совместимых устройств, работающая по типу соединения «точка-точка». В отличие от предыдущих решений, не является шиной как таковой, а обеспечивает последовательное соединение устройств во внутренней сети шины на скоростях до 16 гигатранзакций в секунду (циклов передачи информационных пакетов) [4]. Соответственно, аппаратная и программная настройка шины PCI-е может быть даже более трудоёмким процессом, нежели отладка спектрометрического оборудования. Однако, данная шина позволяет устройствам обмениваться информацией на огромной скорости практически без непосредственного участия центрального процессора, допускает горячую замену оборудования и его автоматическую настройку.

Шина USB. Стандартная универсальная последовательная шина для любых электронных устройств с контроллером USB. Спецификация версии 2.0 описывает протокол передачи данных на скоростях до 480 МБ/с [3], однако время отклика оборудования зависит от многих факторов. Оборудование также нуждается в отдельном контроллере USB. В связи с возможной логической сложностью устройства USB-сети (соединенные контроллеры, хабы, функции) программирование драйвера и аппаратное управление устройством может оказаться затруднено сложной логикой доставки и обработки сигналов и недетерминированными временными задержками [5], зависящими от узлов, соединяющих спектрометрическое оборудования и обрабатывающий процессор. Нетрудно заметить, что данный метод лучше подходит для внешнего спектрометрического комплекса.

Протокол Ethernet. Стандартный сетевой протокол, в большинстве своём использующий технологии IP/TCP для организации взаимодействия узлов и обеспечения высокой скорости передачи данных (до 1 Гбит/с) при достаточно высоком времени отклика сети и физической удалённости её узлов [6]. Данная технология не является достаточно подходящей для аппаратной реализации в виде отдельного АЦП, т.к. не только требует наличия специализированного сетевого оборудования, но также и не даёт возможности для своевременной обработки пришедших сигналов. Однако, её можно использовать в удалённых спектрометрических системах ввиду масштабной унификации протокола и поддержки со стороны операционных систем любого уровня.

Естественно, что аппаратное управление зависит не только от типа компьютерной шины, но и от программных ресурсов вычислительной системы и алгоритмов обработки данных. Так, при решении задачи управления спектрометрическим оборудованием можно рассмотреть следующие варианты организации аналого-цифрового преобразователя:

1) организация спектрометрического АЦП, напрямую подключённого к простой параллельной шине и 
делегирование вопросов арбитража и интерфейса взаимодействия аппаратному и программному обеспечению подключенного компьютера;

2) использование программируемой логической матрицы (ПЛМ), в этом случае, аппаратное управление сводится к автоматическому считыванию данных с АЦП по готовности и их пересылке на соответствующий контроллер шины;

3) использование программируемого микроконтроллера для организации интеллектуального управления спектрометрическим АЦП и взаимодействия с обрабатывающим оборудованием.

Как известно, применение ПЛМ для организации контролирующего аппаратного обеспечения во многих случаях может оказаться более выгодным и быстродействующим решением по ряду причин. Во-первых, схемы логической структуры ПЛМ являются более-менее универсальными и не зависят от производителя чипов, в то же время, для использования разнообразных микроконтроллеров необходимо наличие знаний в области конкретной модели микроконтроллера и его логического управления. Соответственно, программы для микроконтроллеров являются в меньшей степени переносимыми между различными моделями или архитектурами. Во-вторых, работа микроконтроллера основывается на обработке машинных инструкций, которые выполняются с различной скоростью и частотой. В то время как в ПЛМ можно задать логику аппаратного взаимодействия в декларативной форме, машинные инструкции имеют более сложную императивную форму, что снижает общую гибкость системы в целом [7].

Также необходимо учитывать и влияние программного окружения при разработке драйверов аппаратного управления спектрометрическим оборудованием на базе IBM-совместимых компьютеров. Есть несколько распространённых подходов:

- написание программ для компьютера в режиме отсутствия окружения (без операционной системы, запуск происходит после начальной загрузки компьютера);

- использование операционных систем, не блокирующих прямой доступ к аппаратному обеспечению (например, DOS);

- использование операционных систем реального времени (ОСРB), например, RTOS32, QNX, VxWorks;

- использование модулей реального времени для операционных систем общего назначения (например, RTX64 для Windows) [8];

- использование операционных систем общего назначения (например, Windows, FreeBSD, Minix).

Естественно, что каждый из подходов обладает своими преимуществами и недостатками. Список составлен в порядке уменьшения предсказуемости и простоты организации аппаратного управления. Очевидно, что квалификация, требуемая от разработчиков системы, тоже разнится в зависимости от выбранных методов. Так, при реализации системы управления без операционной системы, от разработчиков требуется создание программных компонентов для управления не только спектрометрическим оборудованием, но и аппаратными средствами самой рабочей станции, что значительно увеличивает время и стоимость разработки, а также снижает гибкость самой системы. В то же время, использование минимальных окружений, типа системы DOS, хоть и решает проблему абстракции над аппаратными средствами компьютера, но всё же не лишено тех проблем, с которыми придётся столкнуться при разработке без окружения: организация безопасности (как информационной, так и электронной), организация многозадачности, поддержка высокоуровневых инструментов разработки и прочее. Частично данные проблемы могут быть решены с помощью операционных систем реального времени, в которых реализованы средства многозадачности, распределения ресурсов, библиотеки графического интерфейса пользователя и мощная кроссплатформенность между различными архитектурами процессоров. ОСРВ обладают жёсткими ограничениями по предсказуемости времени отклика, и это будут, в большинстве случаев, платные решения, используемые в промышленности, авиации, военной отрасли и в космических программах [8]. Гораздо более экономичным решением может оказаться использование модулей реального времени, встраиваемых в обычные пользовательские операционные системы. Разработка в среде таких модулей требует квалификации в области проектирования систем реального времени, однако также опирается на использование стандартных интерфейсов программирования родительской операционной системы. Так например, квалифицированный разработчик среды Windows способен в короткие сроки научиться использовать модуль реального времени для этой операционной системы, что повышает гибкость и снижает стоимость разработки.

Конечно, просчёт и коррекция проблемы мёртвого времени на таких системах может стать тривиальной задачей, однако ОСРВ являются достаточно специфичными для практической спектрометрии, которая используется не только в промышленных условиях, но и в лабораторных или учебных. В аппаратных решениях, опирающихся на вычислительные устройства пользовательской стороны, не всегда представляется возможным навязывать использование конкретных операционных систем конечными пользователями. В таком случае, необходимо положиться на средства, предоставляемые операционными системами общего назначения. И хорошо, если это будет разработка на уровне ядра в операционной системе Windows, т.к. компания Microsoft прилагает огромные усилия для сохранения обратной совместимости компонентов. B мире Linux ситуация несколько иная: совместимости между приложениями уровня ядра, предназначенными для различных дистрибутивов, практически нет, равно как и между версиями одного и того же ядра. Поэтому от разработчиков 
могут потребоваться обширные знания и высокий уровень квалификации в области целевых операционных систем для достижения наиболее широкого охвата аудитории, что естественным образом увеличивает сложность и стоимость разработки. Ко всему прочему, при использовании современных операционных систем, всегда необходимо наличие совместимых контроллеров шин на соответствующем спектрометрическом оборудовании, будь то непосредственно АЦП, либо же интегрированная спектрометрическая система - это упрощает использование стандартных шин и обеспечивает взаимодействие с целевой рабочей станцией, поскольку современные операционные системы блокируют доступ к нестандартным аппаратным ресурсам (разработка уровня ядра может многократно усложниться).

\section{ПРИМЕРЫ РЕАЛИЗАЦИИ СПЕКТРОМЕТРИЧЕСКОГО ОБОРУДОВАНИЯ}

В случае создания интегрированной спектрометрической системы, вопросы, касающиеся взаимодействия с компьютером, решаются использованием стандартных шин, описанных выше, а аппаратная реализация вычислительного ядра может быть также основана на ПЛМ, отдельном микроконтроллере или же на базе промышленного компьютера, подключённого к АЦП (в таком случае, проблемы аппаратного взаимодействия с АПЦ переносятся на соответствующий модуль). Очевидно, что разработка интегрированной системы - это более сложный и трудоёмкий процесс, как с производственной точки зрения, так и программной реализации необходимой логики, однако он является более перспективным вариантом в плане удобства для конечного пользователя. На практике же существуют реализации как в виде интегрированных систем, использующих для передачи данных и удалённого управления различные интерфейсы, так и в виде отдельных спектрометрических АЦП, подключаемых к рабочей станции. Рассмотрим для примера некоторые реальные системы.

\section{Спектрометрические АЦП фирмы «Аспект».}

- АЦП-8К-2М - спектрометрический АЦП Вилкинсона, встраиваемый в персональный компьютер на базе 16-разрядной шины ISA. Реализован в виде отдельной платы с 62-пиновым коннектором ISA, встроенным контроллером шины и специализированными интегральными схемами управления. Работает на частоте преобразования 100 МГц. Плата также оборудована встроенной памятью, предназначенной для формирования спектра без непосредственного участия центрального процессора.

- АЦП-8К-В1 - аналогичный предыдущему АЦП, реализованный на базе шины РСІ с более высокой пропускной способностью и временем отклика системы.

- АЦП-USB-8К-В - приборное исполнение спектрометрического АЦП с использованием контроллера USB для удалённого управления с клиентского компьютера [9].

Рассмотренные решения поставляются вместе с управляющим программным обеспечением под систему Windows (SpectraLine) и MS-DOS (An). Драйверы реализуют общение с устройством через стандартизированные используемые шины, а управление осуществляется в виде передачи управляющих команд и считывания данных из встроенного запоминающего устройства. Поскольку время преобразования предложенных АЦП Вилкинсона составляет порядка $3 . .5$ мкс, использовать АЦП напрямую через шину не представляется практичным решением - здесь на помощь приходит встроенное запоминающее устройство (3У). Коррекция мёртвого времени осуществляется электронными средствами, встроенными в управляющие схемы. Одним из примеров можно привести коррекцию мёртвого времени, внесённую временем измерения уровня самим АЦП. Так как время разряда конденсатора известно и зависит от амплитуды пришедшего импульса, вклад в мёртвое время корректируется, исходя из полученного значения с АПЦ, как функция времени от считанной величины.

\section{Спектрометрические системы фирмы «Парсек»}

- 4K-CAA USB анализатор — спектрометрическая система (блок детектирования, ФЭУ и источник питания, накапливающий спектрометрический АЦП), реализущая удалённое управление через интерфейс USB. Характерная особенность - заявленное постоянное мёртвое время системы управления в 1 мкс, что можно легко откорректировать дополнительным временем измерения спектра до требуемой статистической значимости.

- 4К-САЦП-USB - спектрометрический АПЦ Вилкинсона, встроенный в анализатор 4К-САА, и способный питаться от шины USB. Имеет встроенное запоминающее устройство, которое позволяет накапливать и считывать данные без непосредственного участия центрального процессора клиента. Данный АЦП измеряет входные импульсы с длительностью в диапазоне от 0.5 до 20 мкс и имеет время измерения до 5 мкс (настраиваемое). Соответственно, время измерения и выборки корректируется электронными средствами системной платы [9].

Интересным устройством в продукции «Парсек» является модель САЦП-НМВ-16К, реализующая технологию ZDT («нулевое мёртвое время»), разработанную фирмой «ОRTЕС». Данная технология подразумевает коррекцию спектра в реальном времени, обеспечивающую получение спектра, свободного от потерь мёртвого времени. Для достижения этого эффекта, измерительный цикл электронными средствами разбивается на дифференциальные временные интервалы, которые достаточно малы, чтобы скорость счёта не 
успевала ощутимо измениться в течение каждого из этих интервалов. Продолжительность интервала обычно выбирается в диапазоне от 0.1 до 1.5 мс. Во время каждого дифференциального временного интервала измеряется текущее отношение реального времени к живому времени $r=\left(T / T_{Ж}\right)$ [1]. Когда очередной импульс обрабатывается анализатором, вместо добавления единицы в ЗУ соответствующего энергетического канала, значение в этом канале увеличивается на величину $r$, что позволяет мгновенно получать скорректированные отсчёты на каждом анализируемом импульсе.

\section{ЗАКЛЮЧЕНИЕ}

Принимая во внимание указанные материалы и примеры реализаций спектрометрических систем профильными компаниями, можно сделать несколько замечаний и выводов касательно разработки спектрометрического оборудования, и решения проблемы мёртвого времени. Очевидно, что достаточными производственными мощностями для сбора плат АЦП со специализированными интегральными схемами, обладают далеко не все фирмы, занимающиеся разработкой оборудования. Поэтому имеет смысл учесть возможность использования решения проблемы с помощью универсальных систем: программируемых матриц или микроконтроллеров, использование удалённых рабочих станций с операционными системами общего назначения.

Поскольку современные АЦП способны преобразовывать сигналы на достаточно высокой частоте, генерируя полезные данные раз в несколько микросекунд, имеет смысл создавать изначально накапливающий АЦП, чтобы не нагружать системную шину клиентского компьютера. С одной стороны, отсутствие встроенного ЗУ может быть оправдано конструкционной простотой платы АЦП, которая возлагает процесс обработки данных на клиентскую машину, но с другой - ввиду массового использования операционных систем общего назначения, отнимать процессорное время от устройств пользовательского интерфейса и фоновых программ было бы неразумно. Наладить своевременную обработку сигналов под операционной системой общего назначения - процесс трудоёмкий, и данный подход неизбежно будет вносить недетерминированное мёртвое время в измерительный процесс. Как можно заметить, производители профессиональных АЦП используют отдельные модули с запоминающим устройством и контроллером шины, которые позволяют корректировать искажения, связанные с мёртвым временем в детерминированной среде встроенными средствами. Таким образом можно избавиться сразу от трех проблем: от мёртвого времени, вносимого обработкой информации пользовательским устройством, от нагрузки на системную шину компьютера и от необходимости устанавливать особое программное обеспечение на клиентскую машину. Поскольку обновление информации на экране - процесс более медленный, нежели накопление спектрометрической статистики, данные могут передаваться с заметно более низкой частотой (от 15 до 50 мс) и сразу целым блоком.

Когда поднимается вопрос об используемой шине данных, всегда есть выбор между универсальностью и производительностью. Конечно, монтировать USB устройство на клиентской стороне быстрее и проще, но программирование и использование протокола USB (даже современных спецификаций) связано с трудностями излишней сложности и универсальности шины. Действительно, обход дерева устройств в USB-сети может вносить неопределённые временные задержки, что недопустимо для систем, чувствительных ко времени реакции обрабатывающей аппаратуры. В этом плане Ethernet может показывать ещё худшие характеристики, уступая преимущество развитой сетевой структуры (например, в системе может быть множество различных АЦП и детектирующих устройств) скорости обработки информации внутри самой сети. Альтернативой этим вариантам может стать шина PCI Express, предлагая и приемлемую ширину канала, и время отклика, но имея свои сложности с организацией контроллера шины и драйверов устройства. В самом простом варианте же, использование промышленной шины ISA может иметь свои преимущества, так как разработка средств аппаратного управления под ней не осложнена спецификацией и может опираться на определённые соглашения IBM-совместимых компьютеров. В таком случае, обработка сигналов чаще, чем раз в 2-4 мкс не представляется возможной. Также такой подход накладывает ограничение на клиентские операционные системы, ведь в операционных системах общего назначения предоставление необходимых ресурсов для аппаратного управления требовательной ко времени отклика спектрометрической системой через нестандартный интерфейс затруднено.

На основании проведенного анализа особенностей функционирования аппаратных интерфейсов, программной организации работы операционных систем, методов и средств разработки программного обеспечения можно сделать вывод, что одним из наиболее приемлемых вариантов построения систем автоматического контроля и управления процессами реального времени может стать разработка разделенных систем, состоящих из внешнего контроллера и компьютера для обработки и визуализации получаемых данных.

В случае проектирования системы сбора спектрометрической информации на внешний контроллер возлагается задача максимально быстрого реагирования на завершение процесса измерения амплитуды очередного пришедшего от детектора импульса. Контроллер может быть построен как микропрограммный автомат на основе программируемой логической матрицы, так и может быть реализован на основе микроконтроллера или одноплатного компьютера в промышленном исполнении. В последнем случае для 
организации контролирующего ядра предлагается использовать минимальное окружение в виде операционной системы типа DOS, ввиду достаточного уровня абстракции и в то же время свободного доступа к аппаратному обеспечению контроллера. Обработка получаемой информации должна выполняться на этом уровне в минимально необходимом объеме. Программное обеспечение внешнего контроллера может не предусматривать непосредственного подключения к нему средств человеко-машинного интерфейса, а подготавливаемые промежуточные данные измерений (например, массив измеряемого спектра) должны иметь строго нормированный и документированный формат и возможность периодической передачи в обрабатывающий компьютер с использованием одного из универсальных интерфейсов последовательной передачи данных. Такая архитектура системы совмещает в себе плюсы как разработки уровня ядра на контролирующем оборудовании, так и высокоуровневого программирования на уровне клиентской машины, что позволяет создавать развитый графический интерфейс пользователя и использовать вычислительносложные алгоритмы анализа данных. В то же время, использование внешнего устройства обеспечивает гибкость и универсальность при монтировании оборудования на клиентской стороне.

\section{СПИСОК ЛИТЕРАТУРЫ}

1. Gavrilov L.E. Nuclear electronics fundamentals. Part 1. - Moscow: National Research Nuclear University "MEPhI", 2010. P.150, P.154. (in Russian)

2. Shendrik R.I., Radzhabov E.A. Introduction to the physics of scintillators. Part 2. - Irkutsk: ed. IGU, 2014. - 61p. (in Russian)

3. Manoilov V.V. Hardware of Automation Systems for Analytical Devices. - St. Petersburg: National Research University "ITMO", 2012. - P. 72,90,94. (in Russian)

4. Tanenbaum E. Computer architecture. - 5th ed. - St. Petersburg: "Peter", 2010. - P. 232-236, 244-246. (in Russian)

5. Tanenbaum A., Bos H. Modern operating systems (Series "Classic of Computer Science"). - St. Petersburg: Peter, 2015. P. 56-57. (in Russian)

6. Olifer V.G., Olifer N.A. Computer networks. Principles, technologies, protocols: Textbook for high schools -4 th ed. - St. Petersburg: Peter, 2010. - 432p. (in Russian)

7. Parnell K., Roger B. Comparing and Contrasting FPGA and Microprocessor System Design and Development. - XILINX Whitepaper: (WP213 v.1.1), 2004. https://www.xilinx.com/support/documentation/white papers/wp213.pdf

8. Burdonov I.B., Kosachev A.S., Ponomarenko V.N. Real-time operating systems. - Moscow: Institute for System Programming n.a. V.P. Ivannikov RAS, 2006. - P. 3-4, 14-16, 19, 29-31. (in Russian)

9. A review of domestic radiometric and spectrometric systems that can be used for the purposes of nuclear materials accounting and control. [FSE "Russian Scientific Research Institute of Automation named after N.L. Dukhov"] - Moscow. - P. 54-58. (in Russian) 